\title{
Tensile Strength Test of Rock at High Strain Rate Using Digital Image Correlation
}

\author{
Tei Saburi, a ${ }^{1,}$, Yoshiaki Takahashi ${ }^{2, b}$, Shiro Kubota ${ }^{1, c}$ and Yuji Ogata ${ }^{2, d}$ \\ ${ }^{1}$ National Institute of Advanced Industrial Science and Technology, Onogawa 16-1, Tsukuba, \\ Ibaraki 305-8569, JAPAN \\ at.saburi@aist.go.jp, ${ }^{\mathrm{b}}$ takahashi13r@mine.kyushu-u.ac.jp, \\ ckubota.46@aist.go.jp, dyuji-ogata@aist.go.jp
}

\begin{abstract}
Keywords: Tensile Strength, High Strain Rate, Blast Loading, Rock Fracture, Hopkinson Effect, Digital Image Correlation
\end{abstract}

\begin{abstract}
Tensile strength test of rock at high strain rate was experimentally performed by utilizing the nature of the strength difference. A magnitude of the tensile strength of brittle materials such as rock is much smaller than that of compressive strength. A compressive wave was produced by dynamic loading of explosive charge and made incident on a one end of a rock specimen bar. The compressive wave traveled through the specimen bar and it reflected at the free surface of the opposite end as a tensile wave with reversal amplitude. The tensile wave will cause the spall failure of the specimen at a specific distance from the free surface where the superposition of tensile and compressive waves exceeds the tensile failure strength of the specimen, usually referred to as Hopkinson effect. The dynamic behavior was observed at the side face of the bar specimen using a high-speed video camera, and the captured images were used to analyze the surface displacement behavior using a digital image correlation (DIC) technique. Strain and strain rate distributions on the specimen bar during impact loading were evaluated. The relationship between strain rate and dynamic tensile strength was discussed.
\end{abstract}

\section{Introduction}

Dynamic tensile strength is an important factor affecting rock fracturing and fragmentation during blasting operation in quarries and mines. For the dynamic strength test, the Split Hopkinson Pressure Bar (SHPB) is widely applied because of the wide range of strain rate applicability. Regarding the application of the SHPB method to brittle materials, there are many studies [1,2] such as concrete and rock materials for compressive strength. The SHPB method can be applied not only by the indirect tension [3] but also by the direct tension [4] for tensile strength. However, when the sample is rock, the pressure bars sandwiching the sample should be jointed even in the tension state. In the case of rock mass test materials, it is specified or recommended that the sample core diameter is $50 \mathrm{~mm}$ or more in ASTM [5] and $54 \mathrm{~mm}$ or more in ISRM [6] in indirect tension (Brazilian) test to secure the diameter of the material to some content from the presence of crystals and wrinkles. It is necessary to secure the diameter on the side of the incident bar and the transmission bar, and there is a concern that the system as the SHPB test device will become extensive. Therefore, we apply the dynamic tensile strength test using the Hopkinson effect in this study. 


\section{Experiments}

The outline of the test equipment is shown in Fig.1. An explosive is placed on one end face of a cylindrical rock sample with a diameter of $30 \mathrm{~mm}$. An explosive is detonated by the EBW detonator and impact pressure is applied to the sample. Thereby, a compressive stress wave propagates in the sample, and when it reaches the free end on the opposite side of the sample, it is reflected as a tensile stress wave. The area in which the reflected tensile stress wave propagates is an area that mixes with the compression wave that subsequently arrives. The net tensile condition of compressive stress minus tensile stress causes fracture at a point where the sample is sufficient to cleave and the sample leads to spalling. By evaluating the time change of the tensile stress calculated from the position and time of cleavage that occurred at this time and the displacement velocity that can be measured at the free end of the sample, the dynamic tensile strength can be estimated based on the distance and time from the free surface to the fracture surface. The displacement velocity on the free surface is measured by a laser Doppler displacement meter, and the specification of the fracture position and time is analyzed from a high-speed camera image. As the measuring instrument, a laser vibrometer LV-1610 (He-Ne $663 \mathrm{~nm}$ ) from Ono Sokki was used for vibration measurement at the sample end. The high-speed camera SHIMADZU HPV-X (mono, $400 \times 240$ pixel) was used for the displacement measurement on the side generated by the stress wave propagating in the sample by the shock wave. By using two high-speed cameras, threedimensional analysis of displacement is possible, but at present, distortion analysis in one axial direction was performed with one camera. The shooting was performed at a shooting speed of $500,000 \mathrm{fps}$. The stress propagating in the sample is evaluated from the strain information by strain gauge sticking, including the SHPB method. Although strain gauges can track only the time history of local strain at the attachment point, digital image correlation that performs strain measurement in a wide field of view by optical observation with the recent development of digital imaging technology and numerical calculation technology [7]. The scope of application of the Digital Image Correlation (DIC) method has been expanded, and by using a high-speed camera, it is possible to obtain dynamic strain distribution in a high strain range from the full field of view. Although observation using a high-speed camera has been performed in tensile fracture research using the Hopkinson effect [8,9], it has been used for dynamic distortion analysis in the entire field of vision, which is used only for judging the image of a fracture surface There are few examples. The behavior of rock material under dynamic tensile condition is analyzed and the relationship with tensile failure is clarified by vibration velocity measurement on the free surface of the sample by laser vibrometer and DIC strain analysis by high speed camera.

As a sample for the evaluation test, we used Isahaya sandstone produced in Nagasaki Prefecture. Table 1 shows typical mechanical properties. Generally, as in the case of rock materials, the tensile strength is much lower than the compressive strength, and it can be seen that the material is weak in tension. This test method is a test that utilizes the difference in compressive strength and tensile strength characteristic of rock materials.

Table 1 Material properties of Isahaya sandstone

\begin{tabular}{lc}
\hline Properties & Isahaya sandstone \\
\hline Young's modulus, $E[\mathrm{GPa}]$ & 32 \\
Poisson ratio, $v[-]$ & 0.39 \\
Density, $\rho\left[\mathrm{g} / \mathrm{cm}^{3}\right]$ & 2.44 \\
Uniaxial compressive strength, $f_{\mathrm{c}}[\mathrm{MPa}]$ & 182 \\
Splitting tensile strength, $f_{\text {spu }}[\mathrm{MPa}]$ & 9.9 \\
\hline
\end{tabular}




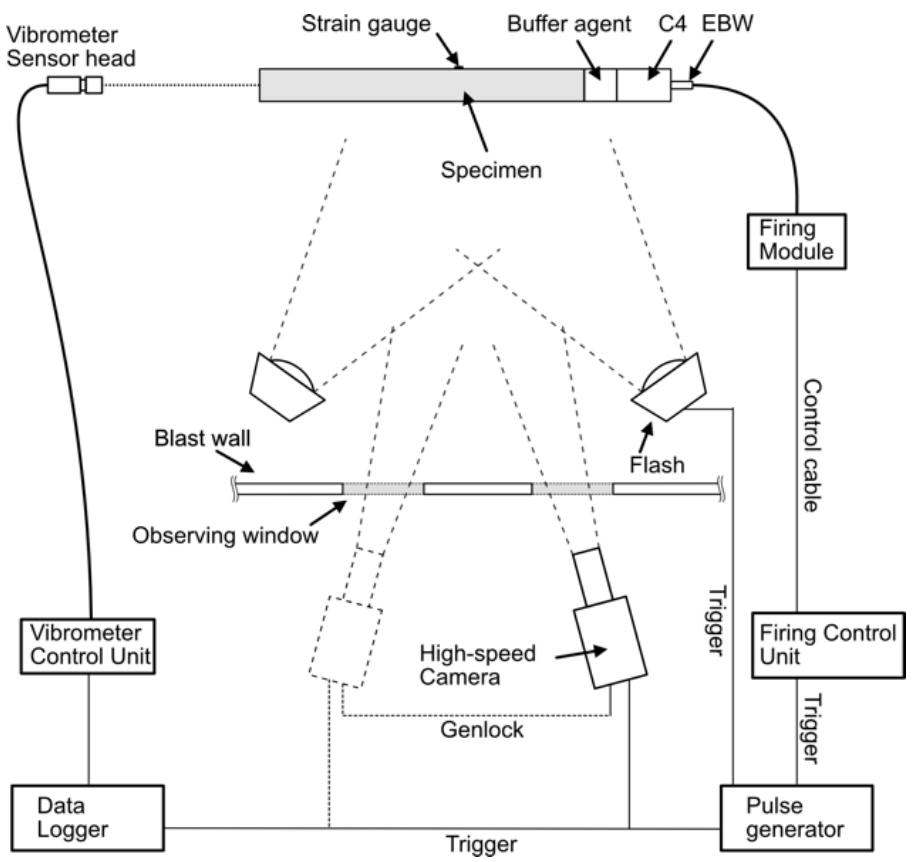

Fig.1 Schematic view of the experimental setup (current system: one-camera for 2D-DIC and no buffer agent).

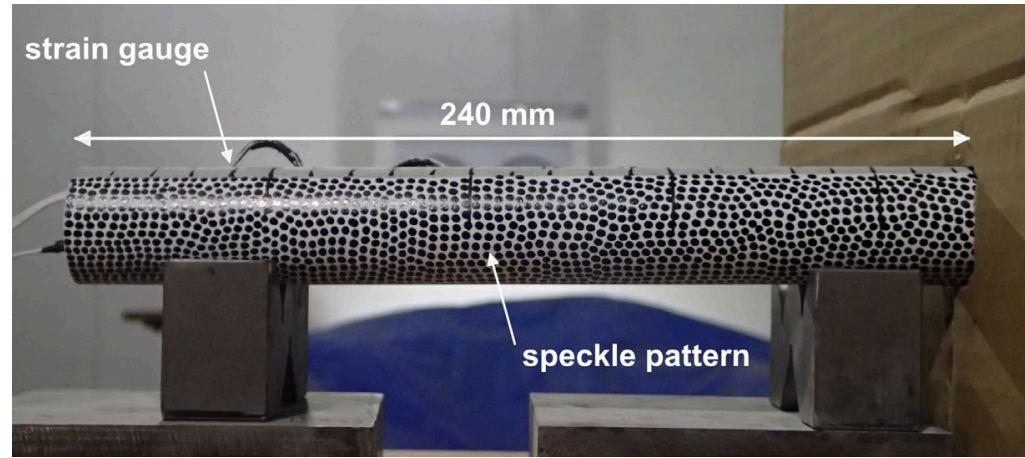

Fig. 2 Picture of sample speciman sprayed speckle patterns for DIC analysis.

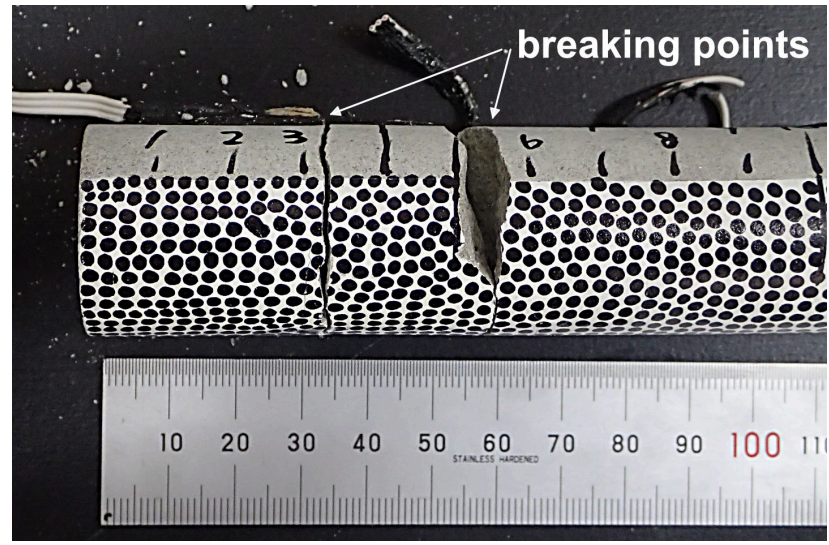

Fig. 3 Picture of sample after the shot.

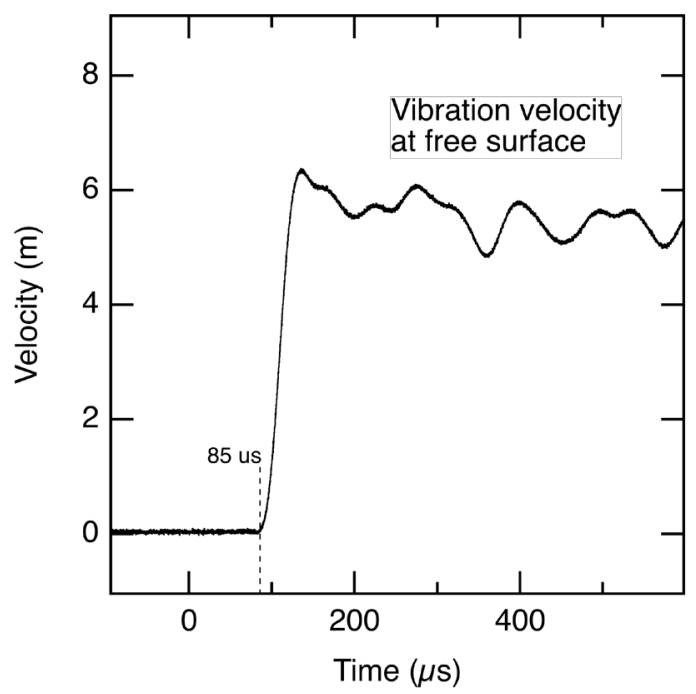

Fig.4 Displacement velocity at free surface. 


\section{Results and Discussion}

The picture of the sample after the shot is shown in Fig. 3. Two spall fractures were identified at 33 $\mathrm{mm}$ and $55 \mathrm{~mm}$ from the free surface. The displacement velocity on the free surface is shown in Fig.4. The wave front reached the free surface after about 85 us, and the maximum displacement velocity thereafter reached about $6.35 \mathrm{~m} / \mathrm{s}$ at about 135 us. The strain distribution in the sample area obtained by the DIC analysis of the captured images of the high-speed camera and the time history for every 10 us of the strain rate distribution are shown in Fig. 5 and Fig. 6, respectively. As a result of DIC analysis, we succeeded in visualizing the expansion and propagation of high strain area along with the propagation of compression wave by impact loading. In addition, it was observed that the compression wave reflected as a tensile wave at the free end, and this caused the strain distribution to be reversed and the fracture to be generated locally at a point where the tensile state became maximum. The strain and strain rate at break point reached up to 0.046 and $50 \mathrm{~s}^{-1}$, respectively. Time histories of line profiles of strain along with the sample were extracted and were shown in Fig.7. It can be confirmed that the tension state starts to be predominant in the region between the two breaking points 95 us after the arrival of the reflected wave at the free surface. Time history of transmitting compression wave front was shown in Fig.8. The propagating velocity of the front was estimated from the slope of the plot and was estimated to be $3315 \mathrm{~m} / \mathrm{s}$, which is $40 \%$ lower than the elastic wave velocity calculated from the reference value shown in Table 1. Peak displacement velocity dU/dt at fracture point was evaluated as $1.83 \mathrm{~m} / \mathrm{s}$ by DIC analysis which is relatively low compared with the half of the peak displacement velocity at free surface assuming free surface reflection as shown Fig. 4.

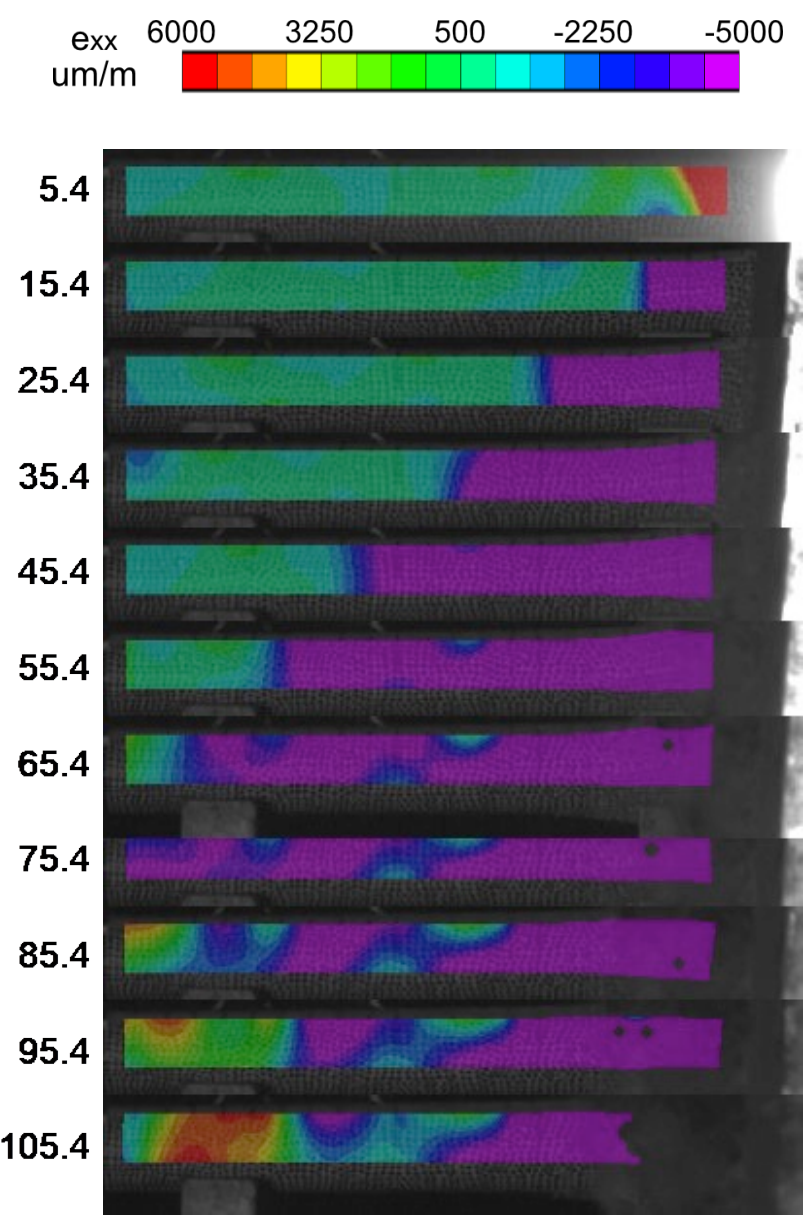

Fig.5 Sequences of strain distribution analyzed by DIC $\left(e_{x x}\right)$
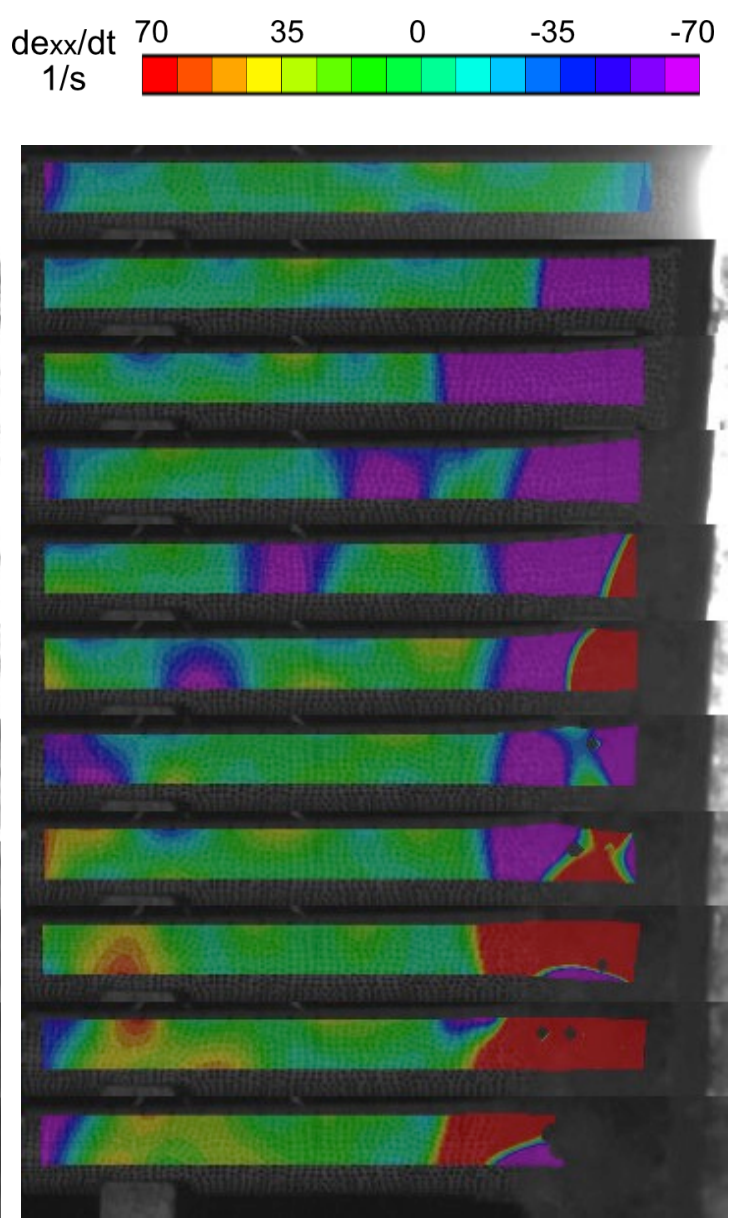

Fig.6 Sequences of strain rate distribution analyzed by DIC $\left(d e_{x x} / d t\right)$ 


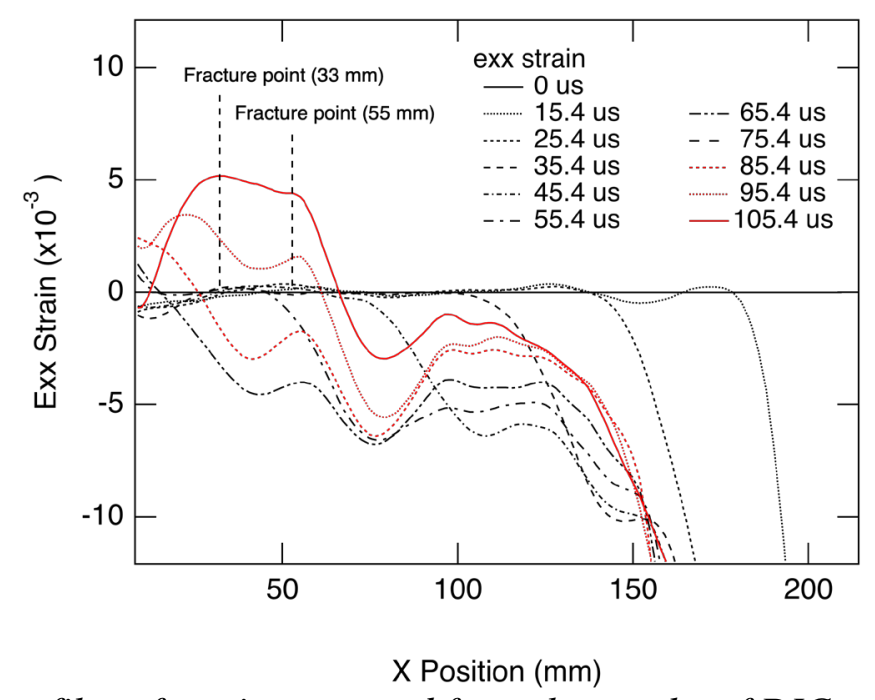

Fig.7 Line profiles of strain extracted from the results of DIC analysis $\left(e_{x x}\right)$.

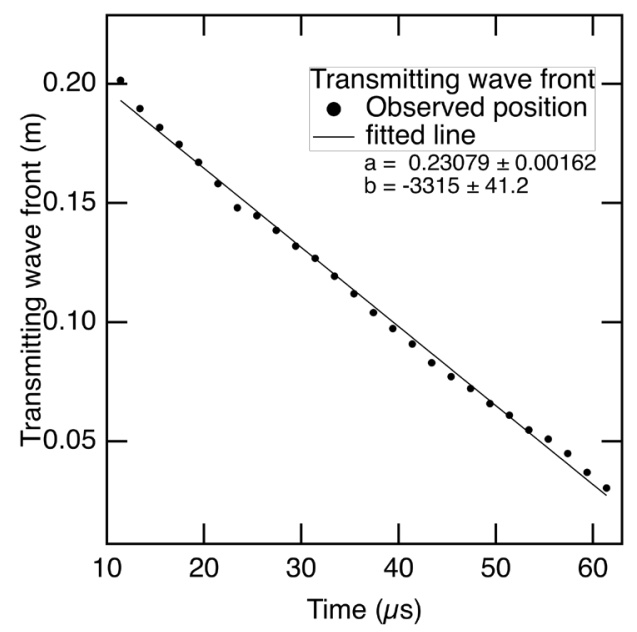

Fig.8 Time history of transmitting compression wave front.

\section{Summary}

In order to clarify the dynamic tensile failure behavior of rock material, dynamic uniaxial tensile experiment by the spalling test under impact loading of explosives based on the Hopkinson effect was proposed and was examined on a sandstone. The dynamic strain distribution and strain rate distribution on the sample were analyzed and visualized by Digital Image Correlation (DIC) method. The expansion and propagation of high strain area along with the propagation of compression wave were successively visualized. It was observed that the compression wave reflected as a tensile wave at the free end, and this caused the strain distribution to be reversed and the fracture to be generated locally at a point where the tensile state became maximum. The strain distribution and strain rate distribution were analyzed by applying DIC method. However, it is necessary to confirm the consistency with values obtained by other measurement methods and calculations. We will continue the experiment by changing the diameter, length and material of the specimen to clarify the relationship among material shapes, strain/strain rate distribution and tensile 
strength and to establish the quantitative estimation method for dynamic tensile strength of rock materials.

\section{References}

[1] C. A. Ross, J. W. Tedesco, S. T. Kuennen, Effects of strain rate on Concrete Strength, ACI Mat. J., 92 (1995) 37-47.

[2] Z. Zhou, X. Li, Z. Ye, K. Liu, Obtaining Constitutive Relationship for Rate-Dependent Rock in SHPB Tests, Rock Mech. Rock Eng., 43 (2010) 697-706. https://doi.org/10.1007/s00603-0100096-3

[3] Q. B. Zhang, J.Zhao, A Review of Dynamic Experimental Techniques and Mechanical Behaviour of Rock Materials, Rock Mech. Rock Eng., 47 (2014) 1411-1478. https://doi.org/10.1007/s00603-013-0463-y

[4] S. Huang, R. Chen, K. W. Xia, Quantification of dynamic tensile parameters of rocks using a modified Kolsky tension bar apparatus, J. Rock Mech. Geotech. Eng., 2 (2010) 162-168. https://doi.org/10.3724/sp.j.1235.2010.00162

[5] ASTM, Standard Test Method for Splitting Tensile Strength of Intact Rock Core Specimens, ASTM D3967-08, (2008). https://doi.org/10.1520/d3967-95ar01

[6] ISRM, Suggested methods for determining tensile strength of rock materials, Int. J. Rock Mech. Min. Sci. Geomech. Abstr., 15 (1978) 99-103.

[7] M. A. Sutton, J. J. Orteu, H. W. Schreier, Image correlation for shape, motion and deformation measurements, Springer (2009). https://doi.org/10.1007/978-0-387-78747-3

[8] SH. Cho, Y. Ogata, K. Kaneko, Strain-rate dependence of the dynamic tensile strength of rock, Int. J. Rock Mech. Min. Sc., 40 (2003) 763-777.

[9] S. Kubota, Y. Ogata, Y. Wada, G.Simangunsong, H. Shimada, K. Matsui, Estimation of dynamic tensile strength of sandstone, Int. J. Rock Mech. Min. Sc., 45 (2008) 397-406. https://doi.org/10.1016/j.ijrmms.2007.07.003 